\title{
Effects of surface site distribution and dielectric discontinuity on the charging behavior of nanoparticles. A grand canonical Monte Carlo study
}

\author{
Received 23rd August 2006, Accepted 25th October 2006 \\ First published as an Advance Article on the web 14th November 2006 \\ DOI: $10.1039 / \mathbf{b 6 1 2 1 1 8 g}$
}

Marianne Seijo, Serge Ulrich, Montserrat Filella, Jacques Buffle and Serge Stoll*

\begin{abstract}
The surface site distribution and the dielectric discontinuity effects on the charging process of a spherical nanoparticle (NP) have been investigated. It is well known that electrostatic repulsion between charges on neighbouring sites tends to decrease the effective charge of a NP. The situation is more complicated close to a dielectric breakdown, since here a charged site is not only interacting with its neighbours but also with its own image charge and the image charges of all its neighbours. Coexistence of opposite charges, titration sites positions, and $\mathrm{pH}$ dependence are systematically studied using a grand canonical Monte Carlo method. A Tanford and Kirkwood approach has been applied to describe the interaction potentials between explicit discrete ampholytic charging sites. Homogeneous, heterogeneous and patch site distributions were considered to reproduce the titration site distribution at the solid/solution interface of natural NPs. Results show that the charging process is controlled by the balance between Coulomb interactions and the reaction field through the solid-liquid interface. They also show that the site distribution plays a crucial role in the charging process. In patch distributions, charges accumulate at the perimeter of each patch due to finite size effects. When homogeneous and heterogeneous distributions are compared, three different charging regimes are obtained. In homogeneous and heterogeneous (with quite low polydispersity indexes) distributions, the effects of the NP dielectric constant on Coulomb interactions are counterbalanced by the reaction field and in this case, the dielectric breakdown has no significant effect on the charging process. This is not the case in patch distributions, where the dielectric breakdown plays a crucial role in the charging process.
\end{abstract}

\section{Introduction}

Many of the important properties of nanoparticle (NP) systems are determined directly or indirectly by the interaction forces between particles. Stability properties of NP dispersions in aqueous solution are mainly governed by their surface charge density, which is determined by the probability of each surface titration site exposing a charged residue. Such a probability depends on chemical equilibrium between a site and the solution which can be tuned by varying the solution $\mathrm{pH}$. In contrast to isolated systems, at the NP surface each titration site is influenced by all other sites via their mutual electrostatic interactions. If electrostatic repulsion between charges on neighbouring sites are expected to decrease the effective NP charge, then the situation is more complicated when a dielectric breakdown is considered, since each charge is interacting with its neighbours but also with its own image charge and the image charges of all its neighbours. Due to the many-body nature of this problem, and the long range influence of the electrostatic interactions, an exact solution for the

CABE (Analytical and Biophysical Environmental Chemistry), Department of Inorganic, Analytical and Applied Chemistry,

University of Geneva, Sciences II, 30 quai E. Ansermet, CH-1211

Geneva 4, Switzerland.E-mail: serge.stoll@cabe.unige.ch calculation of the NP average charge as a function of $\mathrm{pH}$ is generally not known.

The surface charge of NPs in solution has been fully studied by a range of experimental methods, theoretical and numerical models ${ }^{1-6}$ by considering energetically homogeneous surfaces. For an accurate description of the surface charge, a discrete site model has been proposed by Borkovec and co-workers. ${ }^{7,8}$ They consider an Ising model for the solid/solution, a Monte Carlo simulation technique (MC) and a linearized Poisson-Boltzmann approximation with discrete charge descriptions in a planar geometry. ${ }^{9}$

The present work investigates effects of the surface site distribution and the dielectric discontinuity of a spherical $\mathrm{NP}$ on its charging process with solution $\mathrm{pH}$. Due to a spherical geometry, interactions across NPs are also considered. Because of spherical geometry, the Tanford and Kirkwood theory ${ }^{10-12}$ is applied to calculate interaction potentials between charged sites. The $\mathrm{pH}$ influence and the many-body nature of the problem are resolved using MC simulations, according to the Metropolis algorithm. ${ }^{13}$ Simulations have been performed in the grand canonical ensemble (with chemical potential $\mu$, volume $V$, and temperature $T$ fixed) to obtain equilibrium between all the NP charge configurations at a given $\mathrm{pH}$. 


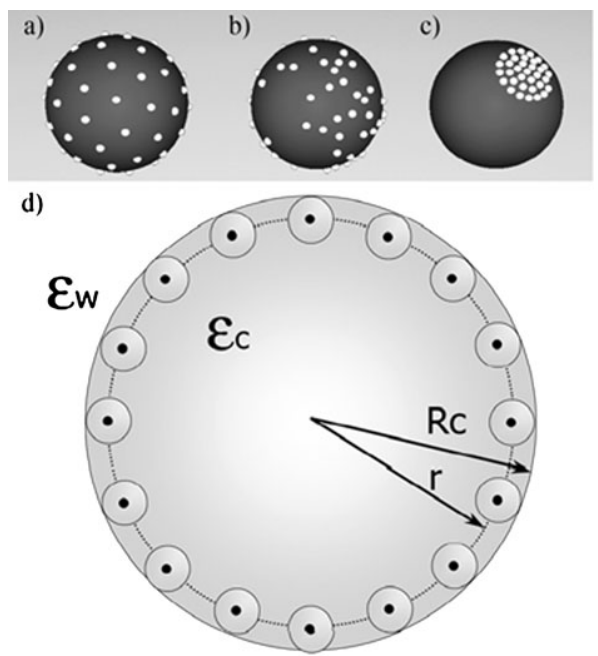

Fig. 1 Spatial configurations of surface discrete charge distributions: (a) homogeneous distribution, (b) heterogeneous distribution and (c) patch distribution. The point-like sites are represented as having a volume on the NP surface to facilitate the representation. (d) A cross section of the NP model. The latter is represented by a sphere surrounded by solution. Titratable sites are represented by fixed point charges located within the spherical dielectric at a distance from the dielectric discontinuity.

The distribution of discrete sites at the NPs may be classified in two categories: a periodic homogeneous discrete site distribution (like a perfect crystal arrangement), or a heterogeneous discrete site distribution. Heterogeneity may originate from defects in crystal planes, disorders in crystal lattices, local chemical impurities, and coexistence of different materials as found in natural solids. ${ }^{14}$ Surface heterogeneity is modeled here using two fundamental distributions: random heterogeneity and clustered, patch distribution. ${ }^{15,16}$ Random heterogeneity occurs either when different types of dissociable functions are available on the surface or when surface passivation is attained. Patch heterogeneity occurs either when different materials coexist or when crystal planes are not large enough to neglect boundary interactions between patches. In this study, homogeneous, heterogeneous and patch distributions of sites are considered (Fig. 1a-c).

The paper is structured as follows: The theoretical model is discussed in section 2. In section 3, a systematic approach is developed in order to quantify interaction potentials and site position influences on the charging process of NPs.

\section{Theoretical modeling}

\subsection{Model description}

The interaction potentials between charged sites have been calculated by considering Tanford's model. ${ }^{10,11}$ The NP is then considered as a sphere in an infinitely diluted aqueous solution, with a continuum representation of the solvent. The sphere is impenetrable to the solvent. In order to take into account the dielectric breakdown, the relative permittivity $\varepsilon_{\mathrm{c}}$ of the NP has to be considered as it can be different from the dielectric constant of the surrounding water $\varepsilon_{\mathrm{w}}$ (Fig. 1d).

Ionizable sites are assumed to be similar in terms of simulation sampling and are represented by fixed point charges located within the spherical dielectric at a distance of $0.5 \AA$ from the dielectric discontinuity $(r=R c-0.5 \AA$, Fig. 1d). This arbitrary distance places charges at about the same position relative to the center of spherical atoms having a diameter of $1.0 \AA$. This localization of sites is not a constraint in the model since site positions can be adjusted within the sphere. All functional groups are assumed to be energetically independent, except for electrostatic interactions.

For the Tanford-Kirkwood theory, and given the elementary charge $e\left(1.63 \times 10^{-19} \mathrm{C}\right)$ and the vacuum permittivity $\varepsilon_{0}$ $\left(8.85 \times 10^{-12} \mathrm{C} \mathrm{V}^{-1} \mathrm{~m}^{-1}\right)$, two ionized sites of charge $z_{i}$ and $z_{j}$ located at a radial position $r$, separated by a distance $r_{i j}$ with an angle $\theta_{i j}\left(\right.$ with $\left.\cos \left(\theta_{i j}\right)=1-r_{i j}{ }^{2} / 2 r^{2}\right)$, contribute to an electrostatic potential energy given (in $k_{\mathrm{B}} T$ units) by

$$
E_{\mathrm{tot}}=\frac{z_{i} z_{j} e^{2}}{4 \pi \varepsilon_{0} k_{\mathrm{B}} T}\left(A_{i j}-B_{i j}\right)
$$

where:

(i) $A_{i j}$ is the direct Coulomb contribution and is given by:

$$
A_{i j}=\frac{1}{r_{i j} \varepsilon_{\mathrm{c}}}
$$

(ii) $B_{i j}$ is the reaction field part of the electrostatic potential energy:

$$
B_{i j}=\frac{1}{\varepsilon_{\mathrm{c}} R_{\mathrm{c}}} \sum_{n=0}^{n_{\max }} \frac{\left(\varepsilon_{\mathrm{w}}-\varepsilon_{\mathrm{c}}\right) P_{n}^{0}\left(\cos \theta_{i j}\right)}{\left(\varepsilon_{\mathrm{w}}+\varepsilon_{\mathrm{c}}(n / n+1)\right)}\left(\frac{r^{2}}{R_{\mathrm{c}}^{2}}\right)^{n}
$$

$P_{n}^{0}$ is the ordinary Legendre polynomial of order $n$ and $n_{\max }$ is the maximum number of terms included in the sum. A large number of terms is needed in the ordinary Legendre polynomial for convergence. When charges are close to the dielectric boundary, the number of terms needed for convergence increases. This sum calculation is an important part of the CPU time. With sites located at $0.5 \AA$ from the dielectric discontinuity, an accuracy of about $10^{-5}$ with $n_{\max }=500$ is achieved, which is a reasonable computer time limit with an accurate convergence. It should be kept in mind that the reaction field part of eqn (1) describes the reaction field accounting for induced polarization charges at the dielectric discontinuity. In absence of dielectric discontinuity, $\varepsilon_{\mathrm{c}}=\varepsilon_{\mathrm{w}}$ and $B_{i j}=0$.

In order to validate the computer program, theoretical results for isolated sites and results obtained by Tanford ${ }^{11}$ and Orttung ${ }^{17}$ with simple models were reproduced correctly.

\subsection{Free energy}

Charges originate from the protonation or deprotonation of the functional groups according to the reaction equilibria

$$
\begin{gathered}
\text { Site }-\mathrm{H}_{2}^{+} \stackrel{K_{\mathrm{a} 1}^{0}}{\rightleftarrows} \text { Site }-\mathrm{H}+\mathrm{H}^{+} \\
\text {Site }-\mathrm{H} \stackrel{K_{\mathrm{a} 2}^{0}}{\rightleftarrows} \mathrm{Site}^{-}+\mathrm{H}^{+}
\end{gathered}
$$


where Site is a functional group, $K_{\mathrm{a} 1}^{0}$ and $K_{\mathrm{a} 2}^{0}$ are equilibrium dissociation constants for eqn (4) and (5), respectively. In the present study, we consider a NP where chemical reactions (4) and (5) occur for each ampholytic titration site.

The acid-base properties of an isolated ampholytic site for a monoprotic chemical reaction, in an aqueous medium, are described by the Henderson-Hasselbach equation ${ }^{18,19}$

$$
\mathrm{pH}=\mathrm{p} K_{\mathrm{a} i}^{0} \pm \log _{10}\left(\frac{1-\alpha_{\mathrm{P}}}{\alpha_{\mathrm{p}}}\right)
$$

where the plus sign is used for proton dissociation and the minus sign for proton association. $\alpha_{\mathrm{p}}$ is the degree of protonation of the isolated ampholyte site.

For a diprotic chemical reaction, the Henderson-Hasselbach equation is

$$
2 \mathrm{pH}=\mathrm{p} K_{\mathrm{a} 1}^{0}+\mathrm{p} K_{\mathrm{a} 2}^{0} \pm \log _{10}\left(\frac{1-\alpha_{\mathrm{P}}}{\alpha_{\mathrm{P}}}\right)
$$

In charged systems such as polymers or NPs, the increase of the charge density is made difficult by the presence of shortand long-range interactions compared with isolated sites in solution. ${ }^{20}$ For a monoprotic chemical dissociation, eqn (6) can be rewritten as

$$
\mathrm{pH}=\mathrm{p} K_{\mathrm{a}}^{0}+\log _{10}\left(\frac{1-\alpha_{\mathrm{P}}}{\alpha_{\mathrm{P}}}\right)+\frac{1}{k_{\mathrm{B}} T \ln (10)} \frac{\partial \bar{U}\left(N_{i}\right)}{\partial N_{i}}
$$

where $N_{i}$ is the number of ionized sites, $k_{\mathrm{B}}$ is the Boltzmann constant $\left(k_{\mathrm{B}}=1.38073 \times 10^{-23} \mathrm{~J} \mathrm{~K}^{-1}\right)$, and $\bar{U}\left(N_{i}\right)$ is the average electrostatic energy of the NP (with $N_{i}$ ionized sites).

For a diprotic chemical dissociation, eqn (7) can be rewritten as

$$
\begin{aligned}
2 \mathrm{pH}= & \mathrm{p} K_{\mathrm{a} 1}^{0}+\mathrm{p} K_{\mathrm{a} 2}^{0}+\log _{10}\left(\frac{1-\alpha_{\mathrm{P}}}{\alpha_{\mathrm{P}}}\right) \\
& +\frac{1}{k_{\mathrm{B}} T \ln (10)} \frac{\partial \bar{U}\left(N_{\mathrm{P}}, N_{0}\right)}{\partial \mathrm{N}_{0}}
\end{aligned}
$$

where $N_{0}$ and $N_{\mathrm{p}}$ are the number of charged sites, with $N_{0}$ the number of unprotonated sites and $N_{\mathrm{p}}$ the number of protonated sites.

\subsection{Monte Carlo Metropolis and statistical ensemble}

NP has $N$ ionizable sites corresponding to $3^{N}$ possible charge states. To explore the configuration space, MC-Metropolis ${ }^{13}$

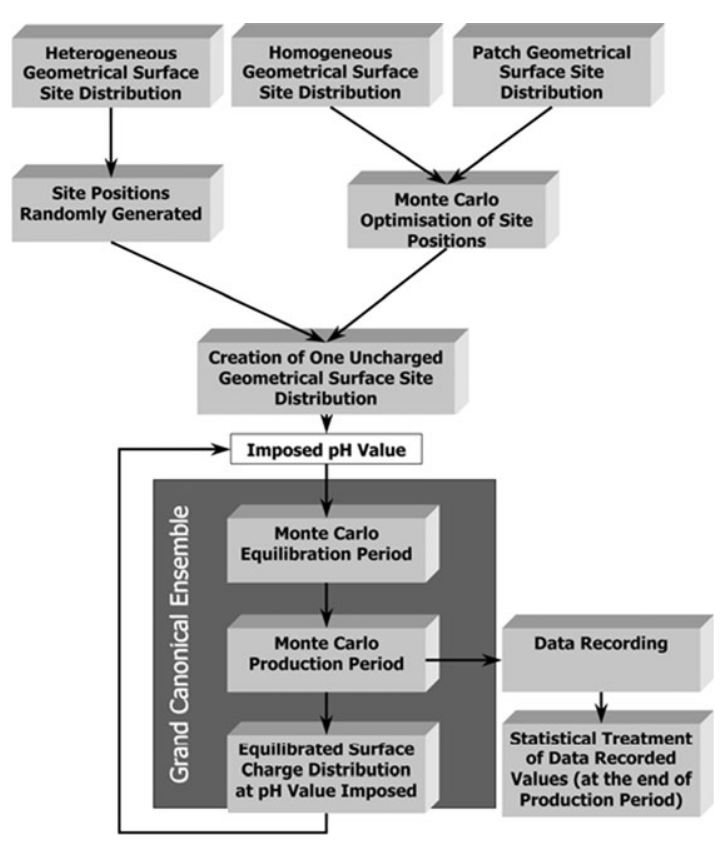

Fig. 2 Flow chart of the MC simulation. Initially a given and uncharged surface site distribution is generated. The $\mathrm{pH}$ value is imposed and a MC equilibration period is achieved without considering any output parameters to avoid any bias due to the initial values and achieve the better level of energy convergence. Then, data recording (energies, charges position, etc.) is made during the MC production period.

simulations are used. For each MC step, a site is chosen at random and its charge state is changed randomly. Two different states are possible among the three possible states (Table 1). Each acid-base reaction is accepted or not by considering the Metropolis selection criterion. The acceptance criterion is sensitive to the value of the energy variation, $\Delta E$, during protonation and deprotonation processes as defined in Table 1. For the sake of clarity, the flow chart of the MC simulation is presented and discussed in Fig. 2.

\subsection{Spatial configuration of surface discrete site distributions}

Spatial distributions of surface discrete titration sites can be classified into three categories: periodic homogeneous discrete site distributions, random heterogeneous discrete site

Table 1 Chemical reactions and associated energy changes

\begin{tabular}{llll}
\hline Initial charge & Possible charge & Acid-base chemical reaction & Change in energy $\Delta E$ (in $k_{\mathrm{B}} T$ units $)$ \\
\hline 0 & +1 & Site $-\mathrm{H}+\mathrm{H}^{+} \stackrel{1 / K_{\mathrm{a} 1}^{0}}{\rightleftarrows}$ Site $-\mathrm{H}_{2}^{+}$ & $\Delta E=\Delta E_{\text {electrostatic }}+\left(\mathrm{pH}-\mathrm{p} K_{\mathrm{a} 1}^{0}\right) \ln (10)$ \\
& -1 & Site $-\mathrm{H} \stackrel{K_{\mathrm{a} 2}^{0}}{\rightleftarrows} \operatorname{Site}^{-}+\mathrm{H}^{+}$ & $\Delta E=\Delta E_{\text {electrostatic }}-\left(\mathrm{pH}-\mathrm{p} K_{\mathrm{a} 2}^{0}\right) \ln (10)$ \\
+1 & 0 & Site $-\mathrm{H}_{2}^{+} \stackrel{K_{\mathrm{a} 1}^{0}}{\rightleftarrows} \operatorname{Site}-\mathrm{H}+\mathrm{H}^{+}$ & $\Delta E=\Delta E_{\text {electrostatic }}-\left(\mathrm{pH}-\mathrm{p} K_{\mathrm{a} 1}^{0}\right) \ln (10)$ \\
& -1 & Site $-\mathrm{H}_{2}^{+} \stackrel{K_{\mathrm{a} 1}^{0} K_{\mathrm{a} 2}^{0}}{\rightleftarrows} \operatorname{Site}^{-}+2 \mathrm{H}^{+}$ & $\Delta E=\Delta E_{\text {electrostatic }}-\left(2 \mathrm{pH}-\mathrm{p} K_{\mathrm{a} 1}^{0}-\mathrm{p} K_{\mathrm{a} 2}^{0}\right) \ln (10)$ \\
-1 & 0 & $\operatorname{Site}^{-}+\mathrm{H}^{+} \stackrel{1 / K_{\mathrm{a} 2}^{\rightleftarrows}}{\rightleftarrows} \operatorname{Site}-\mathrm{H}$ & $\Delta E=\Delta E_{\text {electrostatic }}+\left(\mathrm{pH}-\mathrm{p} K_{\mathrm{a} 2}^{0}\right) \ln (10)$ \\
& +1 & $\operatorname{Site}^{-}+2 \mathrm{H}^{+} \stackrel{1 /\left(K_{\mathrm{a} 1}^{0} K_{\mathrm{a} 2}^{0}\right)}{\rightleftarrows} \operatorname{Site}-2 \mathrm{H}_{2}^{+}$ & $\Delta E=\Delta E_{\text {electrostatic }}+\left(2 \mathrm{pH}-\mathrm{p} K_{\mathrm{a} 1}^{0}-\mathrm{p} K_{\mathrm{a} 2}^{0}\right) \ln (10)$
\end{tabular}


Table 2 Details of the patch distribution position at the NP surface. The total number of sites is 66 in all cases. These 66 sites are identical and homogeneously distributed in the patches. The patch radius is calculated in order to have the same local density per patch, equal to 2.6 sites $\mathrm{nm}^{-2}$

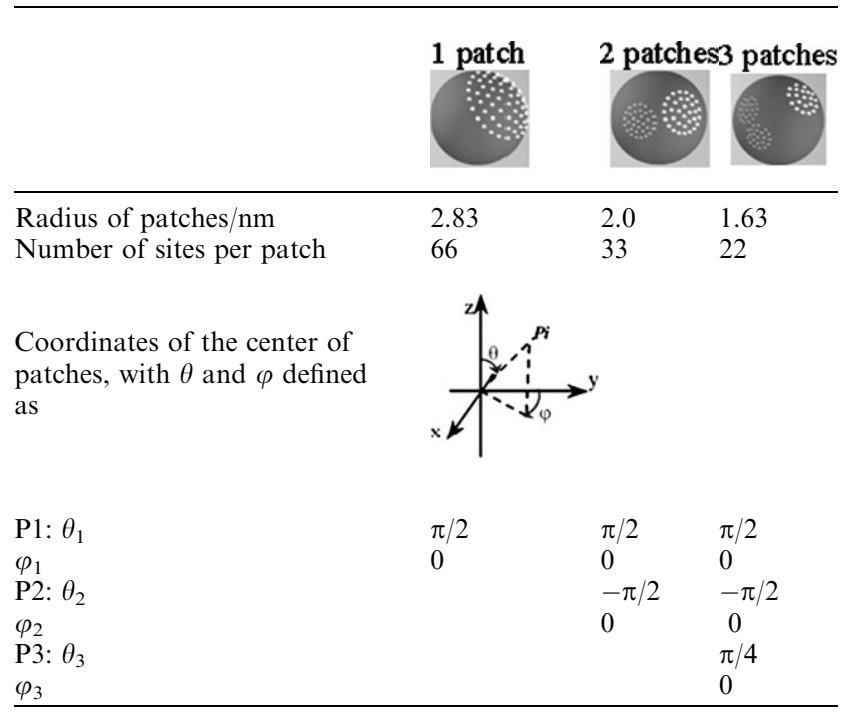

distributions and discrete distributions where sites are confined in patches.

To achieve a homogeneous site distribution, the following procedure is used: First, all sites have the same charge. Then, their positions are adjusted according to the MCMetropolis method to obtain an optimal arrangement of positions among sites. Coordinates of a homogeneous distribution of titration sites are then achieved (Fig. 1a). The problem of optimally arranging charges on the surface of a sphere is a long-standing problem that goes back to the Thomson's plum pudding model of the atom. ${ }^{21}$ Although the Thomson's problem appears simple, its solution still remains difficult. ${ }^{22-24}$

The heterogeneous discrete charge distribution is obtained by randomly distributing sites at the surface of the NP (Fig. 1b).

To obtain patch distributions, titration sites are confined in circular areas with a homogeneous distribution (Fig. 1c). One, two and three patch distributions are specifically studied in the present work.

\section{Results and discussion}

A spherical NP with a radius of $5.0 \mathrm{~nm}$ is considered. The detailed size and positions of the patches are given in Table 2. For all surface site distributions, 66 titration sites are considered arbitrarily, which is a good compromise according to the CPU time. MC equilibration and production times are performed for each $\mathrm{pH}$ value.

The effect of Coulomb interactions is discussed first for each surface site distribution, followed by the effect of the dielectric breakdown.

For the sake of clarity, titration curves are compared with the corresponding ideal cases, i.e. in absence of electrostatic interactions between sites. The relative concentration of deprotonated sites $P_{-1}$ can be written as

$$
P_{-1}=\frac{\left[\text { Site }^{-}\right]}{\left[\text {Site }^{-}\right]+[\text {Site }-\mathrm{H}]+\left[\text { Site }-\mathrm{H}_{2}^{+}\right]}
$$

or

$$
P_{-1}=\frac{1}{1+\left(K_{\mathrm{a} 2}^{0}\right)^{-1}\left[\mathrm{H}^{+}\right]+\left(K_{\mathrm{a} 1}^{0} K_{\mathrm{a} 2}^{0}\right)^{-1}\left[\mathrm{H}^{+}\right]^{2}}
$$

Similarly, for neutral sites it can be written as

$$
P_{0}=\frac{\left(K_{\mathrm{a} 2}^{0}\right)^{-1}\left[\mathrm{H}^{+}\right]}{1+\left(K_{\mathrm{a} 2}^{0}\right)^{-1}\left[\mathrm{H}^{+}\right]+\left(K_{\mathrm{a} 1}^{0} K_{\mathrm{a} 2}^{0}\right)^{-1}\left[\mathrm{H}^{+}\right]^{2}}
$$

and for protonated sites

$$
P_{1}=\frac{\left(K_{\mathrm{a} 1}^{0} K_{\mathrm{a} 2}^{0}\right)^{-1}\left[\mathrm{H}^{+}\right]^{2}}{1+\left(K_{\mathrm{a} 2}^{0}\right)^{-1}\left[\mathrm{H}^{+}\right]+\left(K_{\mathrm{a} 1}^{0} K_{\mathrm{a} 2}^{0}\right)^{-1}\left[\mathrm{H}^{+}\right]^{2}}
$$

For a given $\mathrm{pH}$, the value of the total charge $Z_{\text {tot }}$ of NP is given by (in valence number)

$$
Z_{\text {tot }}=N_{\text {sites }}\left(P_{1}-P_{-1}\right)
$$

Two sets of $\mathrm{p} K_{\mathrm{a}}$ values will be used: $\mathrm{p} K_{\mathrm{a} 1}^{0}=3.0$ and $\mathrm{p} K_{\mathrm{a} 2}^{0}=9.0, \mathrm{p} K_{\mathrm{a} 1}^{0}=6.0$ and $\mathrm{p} K_{\mathrm{a} 2}^{0}=8.0 . \Delta \mathrm{p} K_{\mathrm{a}}$ is defined as $\mathrm{p} K_{\mathrm{a} 2}^{0}-\mathrm{p} K_{\mathrm{a} 1}^{0}$.

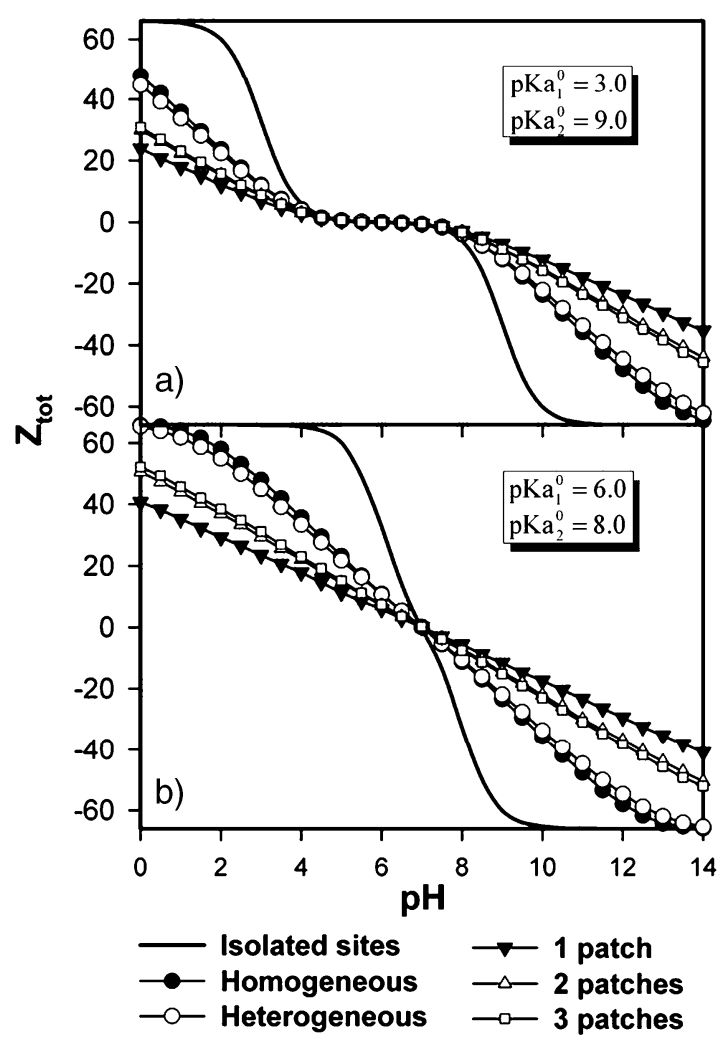

Fig. 3 Effects of Coulomb interactions on the total charge of NP with a radius of $5.0 \mathrm{~nm}$ and 66 titration sites at different site distributions $\left.\varepsilon_{\mathrm{c}}=\varepsilon_{\mathrm{w}}=78.54\right)$ at $T=298 \mathrm{~K}$. 

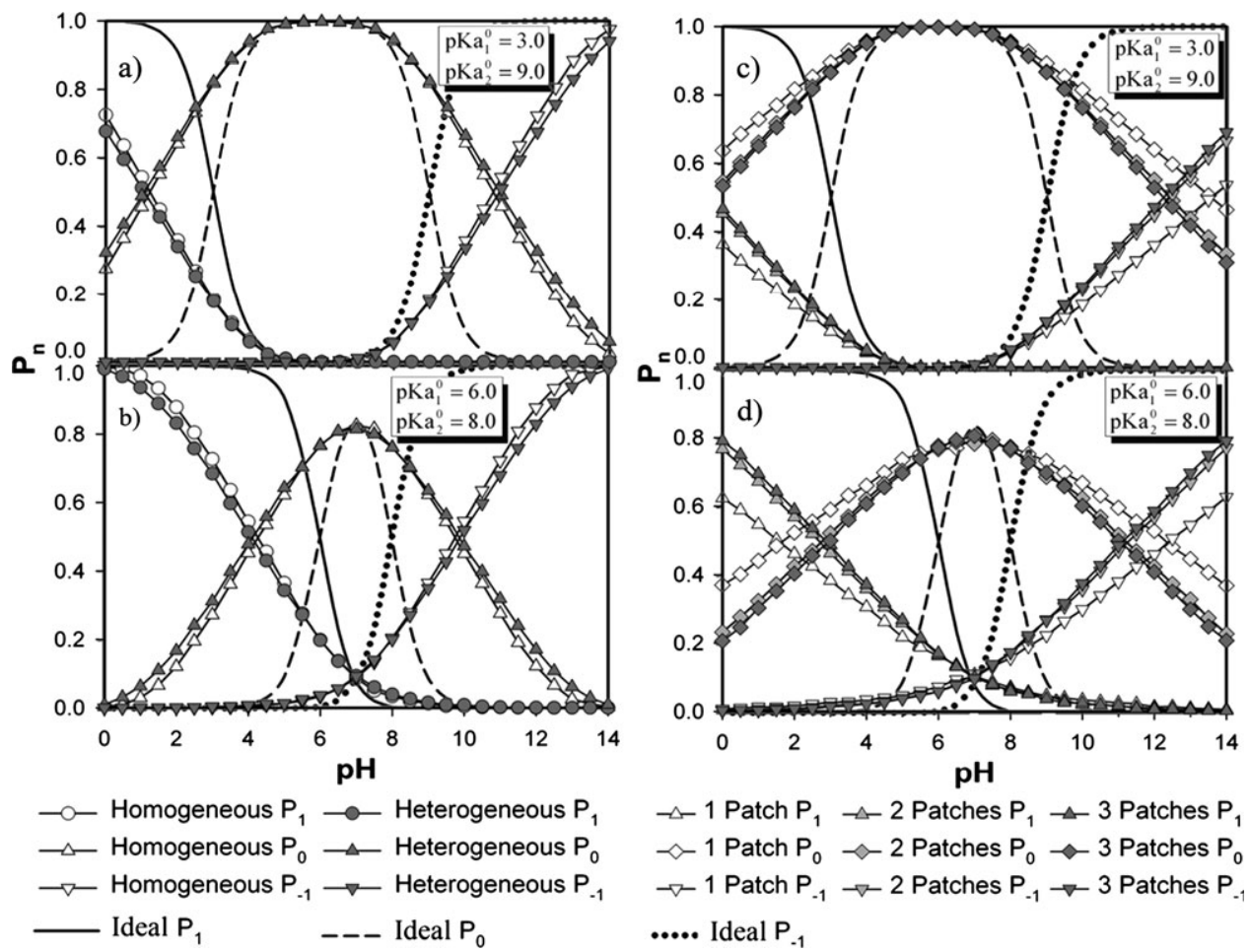

Fig. 4 Effects of Coulomb interactions on the prevalence diagram of NP with a radius of $5.0 \mathrm{~nm}$ and 66 titration sites, with $\varepsilon_{\mathrm{c}}=\varepsilon_{\mathrm{w}}=78.54$ at $T=298 \mathrm{~K}$ for homogeneous and heterogeneous distributions (a) and (b), and for patch distributions (c) and (d). Curves are compared with the corresponding ideal cases, i.e. in the absence of electrostatic interactions between sites.

\subsection{Coulomb interactions}

Simulations are first carried out without any dielectric discontinuity $\left(\varepsilon_{\mathrm{c}}=\varepsilon_{\mathrm{w}}=78.54\right)$ at $T=298 \mathrm{~K}$. Variations of the total charge of the sphere $Z_{\text {tot }}$ as a function of $\mathrm{pH}$ for the two sets of $\mathrm{p} K_{\mathrm{a}}$ and for different site distributions are shown in Fig. 3.

For a given $\mathrm{pH}$, non ideal cases always yield less charges than the ideal case due to the presence of short- and longrange electrostatic repulsions between charged sites, which makes a further increase in the charge density difficult. These results are in good agreement with effects observed by Overbeck $^{20}$ with short- and long-range electrostatic repulsion in a polyelectolyte system.

During the charging process, $Z_{\text {tot }}$ of homogeneous and heterogeneous distributions are very close and patch distributions are less charged, with the one patch distribution being more difficult to ionize.

To get a better insight, prevalence diagrams and energy curves for each site distribution have been investigated (Fig. 4). Prevalence diagrams show that there is no coexistence of positive and negative charges when $\Delta \mathrm{p} K_{\mathrm{a}}=6$. However when $\Delta \mathrm{p} K_{\mathrm{a}}=2$, a small amount of both positive and negative charges is obtained. The coexistence of positive and negative charges in patch distributions is observed over a wider $\mathrm{pH}$ range ( $\mathrm{pH}=2.0-12.0)$ than for the homogeneous and heterogeneous distributions ( $\mathrm{pH}=5.0-9.0)$. This is due to the higher local surface site density as compared to homogeneous and heterogeneous distributions.
Comparisons of energy curves for homogeneous and heterogeneous distributions in Fig. 5 show three different zones corresponding to successive charging process steps:

(i) When $\mathrm{p} K_{\mathrm{a} 1}^{0}<\mathrm{pH}<\mathrm{p} K_{\mathrm{a} 2}^{0}$ (zone A), the total electrostatic energy is close to $0 k_{\mathrm{B}} T$ : there is no difference between homogeneous and heterogeneous distribution titration curves. This is due to the presence of uncharged sites (when $\mathrm{p} K_{\mathrm{a} 1}^{0}=3.0$ and $\mathrm{p} K_{\mathrm{a} 2}^{0}=9.0$ ) or to the coexistence of positively and negatively charged sites (when $\mathrm{p} K_{\mathrm{a} 1}^{0}=6.0$ and $\left.\mathrm{p} K_{\mathrm{a} 2}^{0}=8.0\right)$.

(ii) When $\left(\mathrm{p} K_{\mathrm{a} 1}^{0}-\delta\right)<\mathrm{pH}<\mathrm{p} K_{\mathrm{a} 1}^{0}$ or $\mathrm{p} K_{\mathrm{a} 2}^{0}<\mathrm{pH}<$ $\left(\mathrm{p} K_{\mathrm{a} 2}^{0}+\delta\right)$ (zone B), the energy of the heterogeneous distribution becomes lower than the homogeneous distribution. $\delta$ represents a critical transition parameter which is expected to be dependent on the site positions for the heterogeneous distribution. In this case, $\delta$ is equal to 4.5 . The total charge $Z_{\text {tot }}$ of the heterogeneous distribution is smaller than in the homogeneous distribution in zone B. Due to the site-to-site distance distribution and electrostatic repulsion minimization, charges are situated as far as possible from each other as long as $Z_{\text {tot }}$ is low.

(iii) When $\mathrm{pH}<\left(\mathrm{p} K_{\mathrm{a} 1}^{0}-\delta\right)$ or $\mathrm{pH}>\left(\mathrm{p} K_{\mathrm{a} 2}^{0}+\delta\right)($ zone C), with $\mathrm{pH}$ forcing the charging process. The energy corresponding to the heterogeneous distribution becomes greater than in the homogeneous case (at a given $\mathrm{pH}$ ) since the mean separation distances between sites become smaller.

The critical transition parameter $\delta$ between zones $\mathrm{B}$ and $\mathrm{C}$ is now discussed through the comparison of different heterogeneous configurations and the homogeneous configuration. 

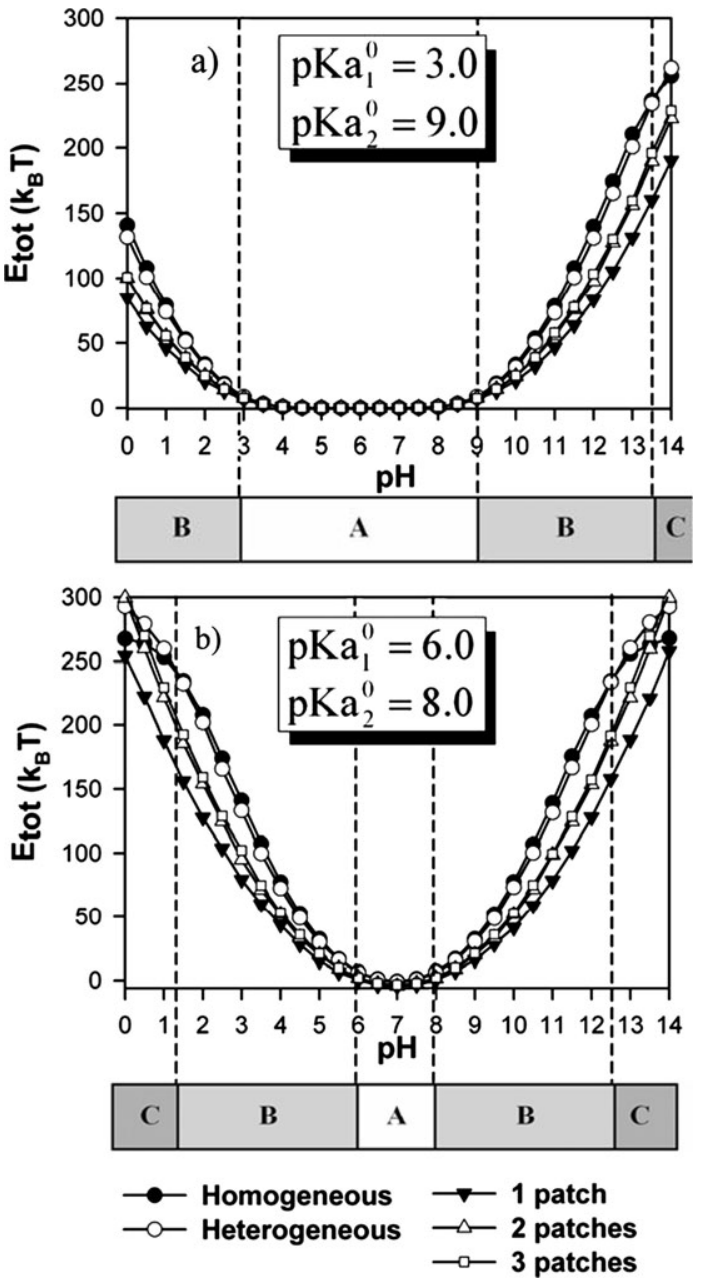

Fig. 5 Effects of Coulomb interactions on the total electrostatic energy for different site distributions versus the $\mathrm{pH}$. Three zones are observed when homogeneous and heterogeneous distributions are compared.

Short- and long-range electrostatic interactions effects on titration curves are quantitatively compared by using a distance polydispersity index, $P_{\mathrm{I}}$, defined as

$$
P_{\mathrm{I}}=\frac{\sum_{j} N_{j} \sum_{j} N_{j} d_{j}^{2}}{\left(\sum_{j} N_{j} d_{j}\right)^{2}}
$$

where $N_{j}$ and $d_{j}$ are the distance number and distance value, respectively. The polydispersity index exhibits lower values when the distance distribution is regular. Calculations were performed for different heterogeneous distributions with $P_{\mathrm{I}}$ equal to $1.1515,1.1378,1.1305$ and 1.1220 .

Polydispersity affects the variation of the total electrostatic energy with $\mathrm{pH}$, as shown in Fig. 6a, and influences the position of the critical transition parameter $\delta$. In zone B for charged configurations, the lower the dispersity index, the higher the total electrostatic energy. In zone $\mathrm{C}$, the inverse is observed: the higher the polydispersity index, the higher the total electrostatic energy. Energy differences are mainly due to
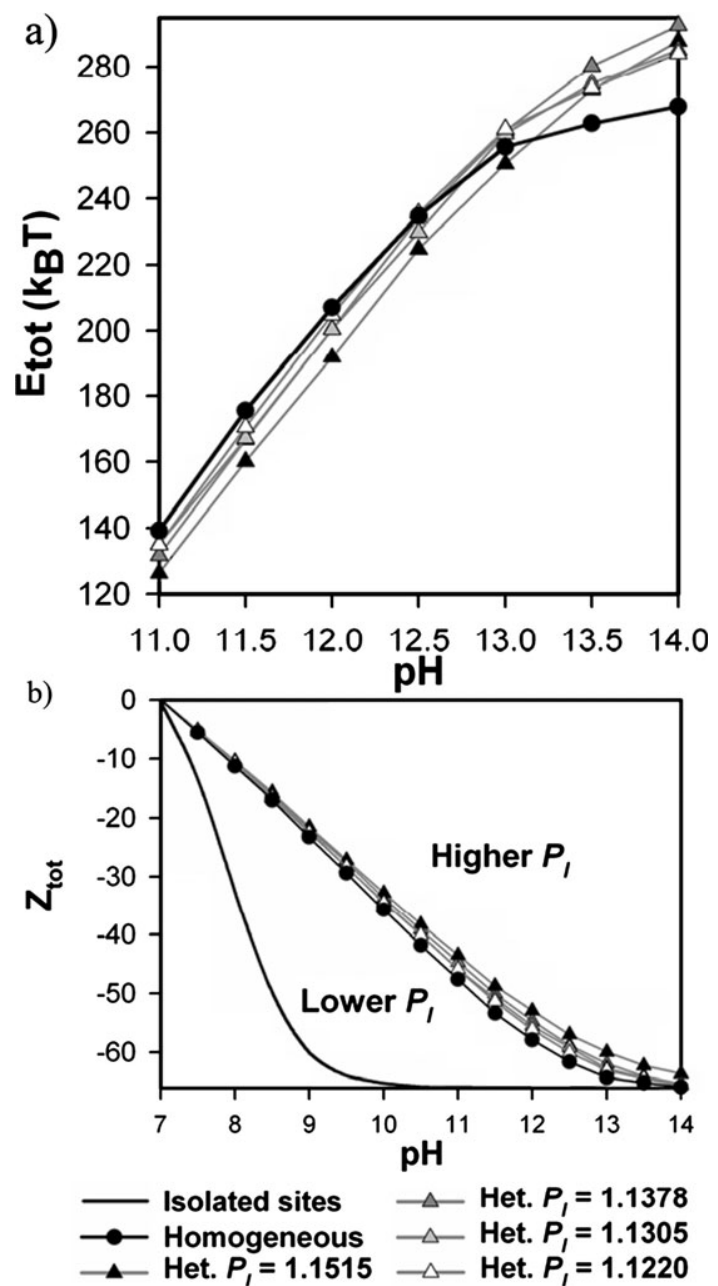

Fig. 6 Effects of Coulomb interactions for a $5.0 \mathrm{~nm}$ radius NP having 66 titration sites with $\mathrm{p} K_{\mathrm{a} 1}^{0}=6.0, \mathrm{p} K_{\mathrm{a} 2}^{0}=8.0$. The results are obtained for homogeneous and several heterogeneous distributions (noted Het. with the corresponding polydispersity index, $P_{\mathrm{I}}$ ) on (a) the total electrostatic energy as a function of $\mathrm{pH}$, in the $\mathrm{pH}$ range 11.0-14.0 in order to observe the critical transition parameter $\delta$ position and (b) the total charge as a function of $\mathrm{pH}$ for a $\mathrm{pH}$ range of 7.0-14.0.

short- (zone C) and long-range (zone B) interaction effects. It should also be noted that when the polydispersity index increases, the NP charging process clearly becomes more difficult as shown in Fig. 6b.

When patch distributions are considered, significant differences in the total charge of the NP as a function of $\mathrm{pH}$ are observed between the single patch distribution, and the two and three patch distributions (Fig. 3). Patch distributions allow qualitative analyses of the charging process in terms of short- (inside patches) and long-range (between patches) electrostatic interactions, which cause the shifts observed in Fig. 3 and 5. Indeed, the number of short-range Coulomb interactions is more important in the one patch configuration compared to the two and three patch configurations. For the three patch distribution, the number of long-range interactions is also more significant than the number of short-range interactions compared to the one and two patch distributions. In the three patch distribution, due to the small separation between 
a)

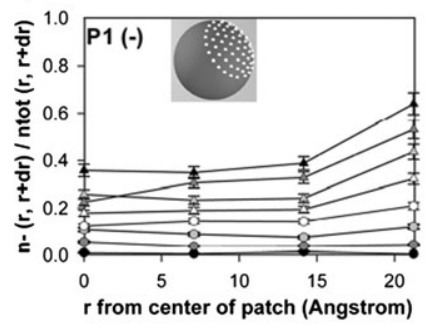

b)

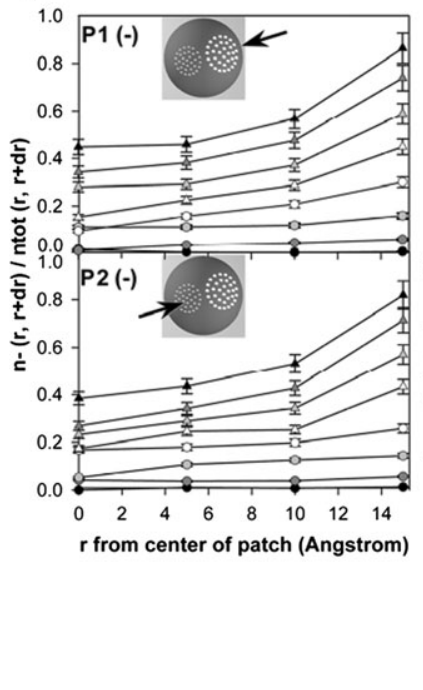

c)

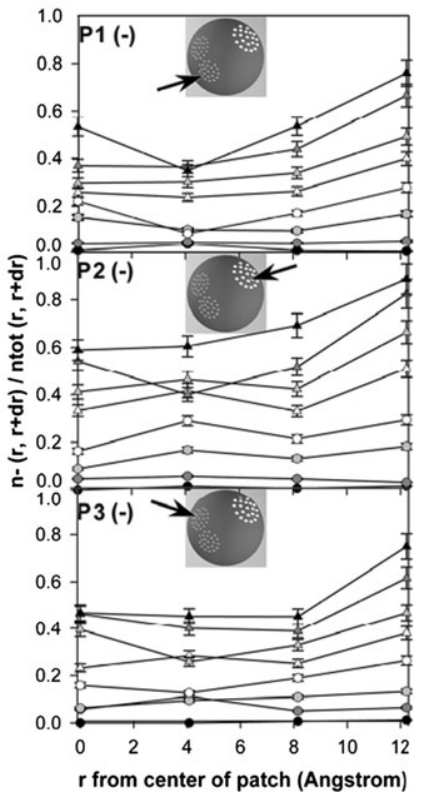

Fig. 7 Radial distribution of negatively charged sites in a finite surface element defined by $r$ to $r+\mathrm{d} r$ as a function of distance $r$ from the center of patch with $\varepsilon_{\mathrm{c}}=\varepsilon_{\mathrm{w}}=78.54$ at $T=298 \mathrm{~K}$ and $\mathrm{p} K_{\mathrm{a} 1}^{0}=3.0$ and $\mathrm{p} K_{\mathrm{a} 2}^{0}=9.0$, for (a) one patch configuration, (b) two patch configuration and (c) three patch configuration as described in Table 2. Arrows indicate the corresponding patch.

patches P1 and P3 (defined in Table 2), the differences between two and three patch configurations are not so significant compared to the one patch configuration where short-range interactions are predominant.

When charge distributions are examined in detail for each patch at different $\mathrm{pH}$ values (Fig. 7), finite size effects are observed. Charge depletion is observed at the center of the patches since the charge accumulates at the perimeter where charge density is lower (due to the presence of less neighboring charge). When patches are close to each other, such as P1 and P3 in the three patch configuration (Fig. 7c), that effect is less marked compared to $\mathrm{P} 2$, which is a more isolated patch.

\subsection{Dielectric discontinuity}

When a dielectric discontinuity between two media (with relative dielectric constants $\varepsilon_{1}$ and $\varepsilon_{2}$ ) is considered, a charge $q$ in medium 1 at a distance $d$ from the dielectric discontinuity is expected to interact with the interface because of the field reflected by this interface. This reaction field is equivalent to an 'image' charge $q^{\prime}$ at a distance $2 d$ from $q .{ }^{25}$ If $\varepsilon_{1}<\varepsilon_{2}$, the resulting reaction field force is attractive and then the $B_{i j}$ term in $E_{\mathrm{el}}$ (eqn (1)) is positive; if $\varepsilon_{1}>\varepsilon_{2}$, the reaction field force is repulsive and the $B_{i j}$ term is negative. In order to investigate the influence of the dielectric discontinuity on the NP charging process, simulations have been carried out with $T=298 \mathrm{~K}$, $\varepsilon_{\mathrm{w}}=78.54$ and by adjusting $\varepsilon_{\mathrm{c}}$ to $2.0,30.0,78.54$ and 114.0.

As illustrated in Fig. 8a, the variation of the total charge of the NP as a function of the $\mathrm{pH}$ and $\varepsilon_{\mathrm{c}}$ shows no significant effect of the dielectric discontinuity for the heterogeneous distribution. The same trends are observed for homogeneous distribution (data are not reported here). In contrast, patch distributions are more affected by $\varepsilon_{\mathrm{c}}$ variations: when $\varepsilon_{\mathrm{c}}$ increases, $Z_{\text {tot }}$ increases due to the decrease in the electrostatic repulsions as shown in Fig. $8 \mathrm{~b}$ for three patch distribution (same trends for one and two patch distributions). In Fig. 8c the relative concentration of deprotonated sites $P_{-1}$ is represented as a function of $\varepsilon_{\mathrm{c}}$. By increasing the NP dielectric constant, the charging process is clearly promoted for all patch distributions. This is due to the importance of the Coulomb contribution on the reaction field.

As shown in Fig. 9 and 10, the total electrostatic energy $E_{\text {tot }}$ is dependent on $\varepsilon_{\mathrm{c}}$ and $\varepsilon_{\mathrm{w}}$, which control both the sign and the intensity of the reaction field part of energy $B_{i j}$ and the amplitude of the electrostatic repulsion through the NP. On the one hand, Coulomb interactions are expected to decrease if $\varepsilon_{\mathrm{c}}$ increases, hence promoting the $Z_{\text {tot }}$ value for a given $\mathrm{pH}$. On the other hand, the reaction field is expected to lower $E_{\mathrm{tot}}$, if $\varepsilon_{\mathrm{c}}<\varepsilon_{\mathrm{W}}$, and hence favor the charging process. Finally, these two competitive effects control the charging process of the NP. At small charge separation distances, the relative importance of the Coulomb interactions is more pronounced than the reaction field interactions. This specifically affects patch distributions, because of the short distances between sites, and explains why $Z_{\text {tot }}$ increases when $\varepsilon_{\mathrm{c}}$ increases.

Modification of the critical transition parameter $\delta$ with $\varepsilon_{\mathrm{c}}$ is not relevant (Fig. 10), because there is no significant effect of the dielectric discontinuity on the homogeneous and heterogeneous distributions.

It should be noted that finite size effects are still observed when the dielectric discontinuity effect on charge positions in patch distributions are considered (data not reported) and comparison with Fig. $7 \mathrm{~b}$ is made. It should be noted that the finite size effect is not affected by $\varepsilon_{\mathrm{c}}$.

\section{Conclusions}

Interactions between charged sites on a spherical nanoparticle (NP) as well as the NP charging process were simulated using a 


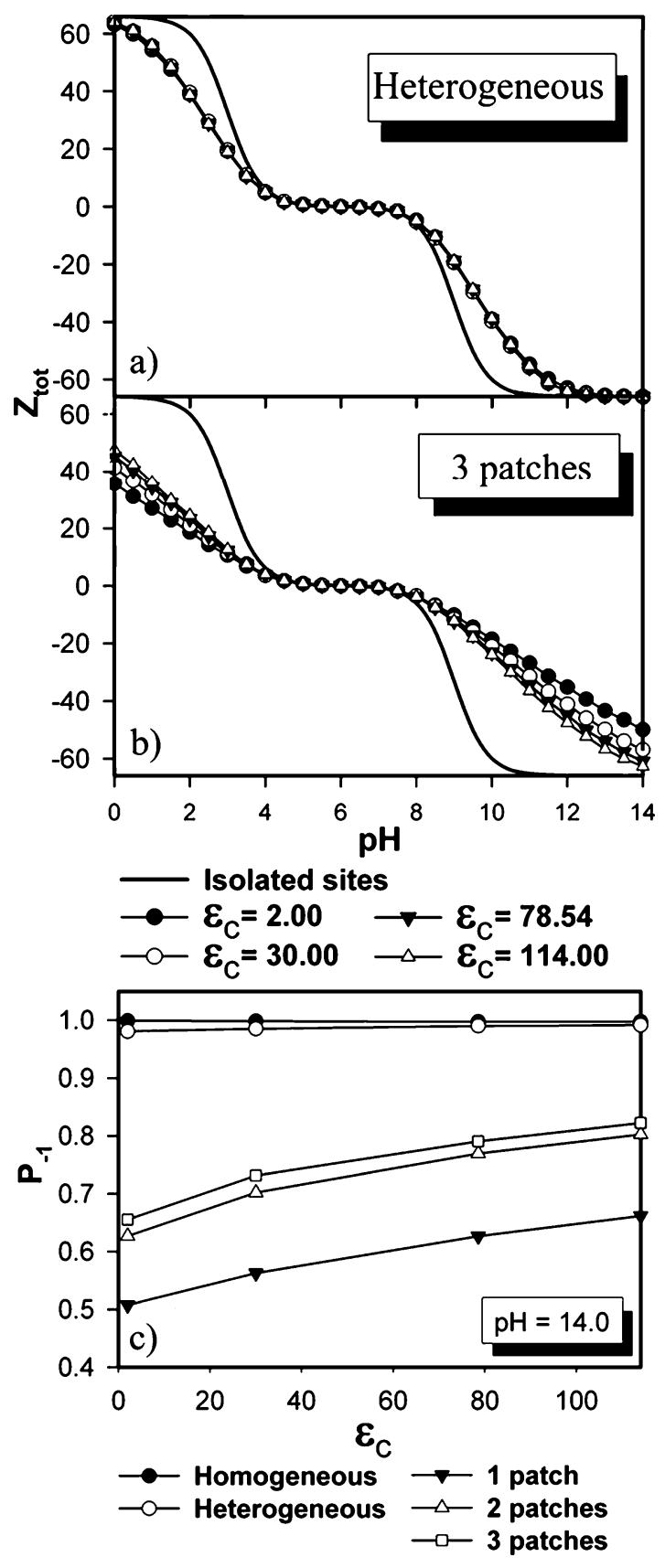

Fig. 8 Effects of Coulomb and reaction field interactions on the total charge $Z_{\text {tot }}$ of NP with a radius of $5.0 \mathrm{~nm}$ and 66 titration sites for different NP permittivities with $\mathrm{p} K_{\mathrm{a} 1}^{0}=6.0, \mathrm{p} K_{\mathrm{a} 2}^{0}=8.0$ for (a) heterogeneous site distributions and (b) three patch site distributions. (c) The relative concentration of deprotonated sites $P_{-1}$ as a function of $\varepsilon_{\mathrm{c}}$ for different site distributions in the same conditions at $\mathrm{pH}=14.0$.

grand canonical MC method that included theoretical extensions in order to take ampholytic sites into account. A Tanford and Kirkwood approach was used to consider spherical geometry and dielectric breakdown.

Because of an explicit description of titration sites, comparisons between different spatial distributions of surface discrete sites (homogeneous, heterogeneous and patch distributions)

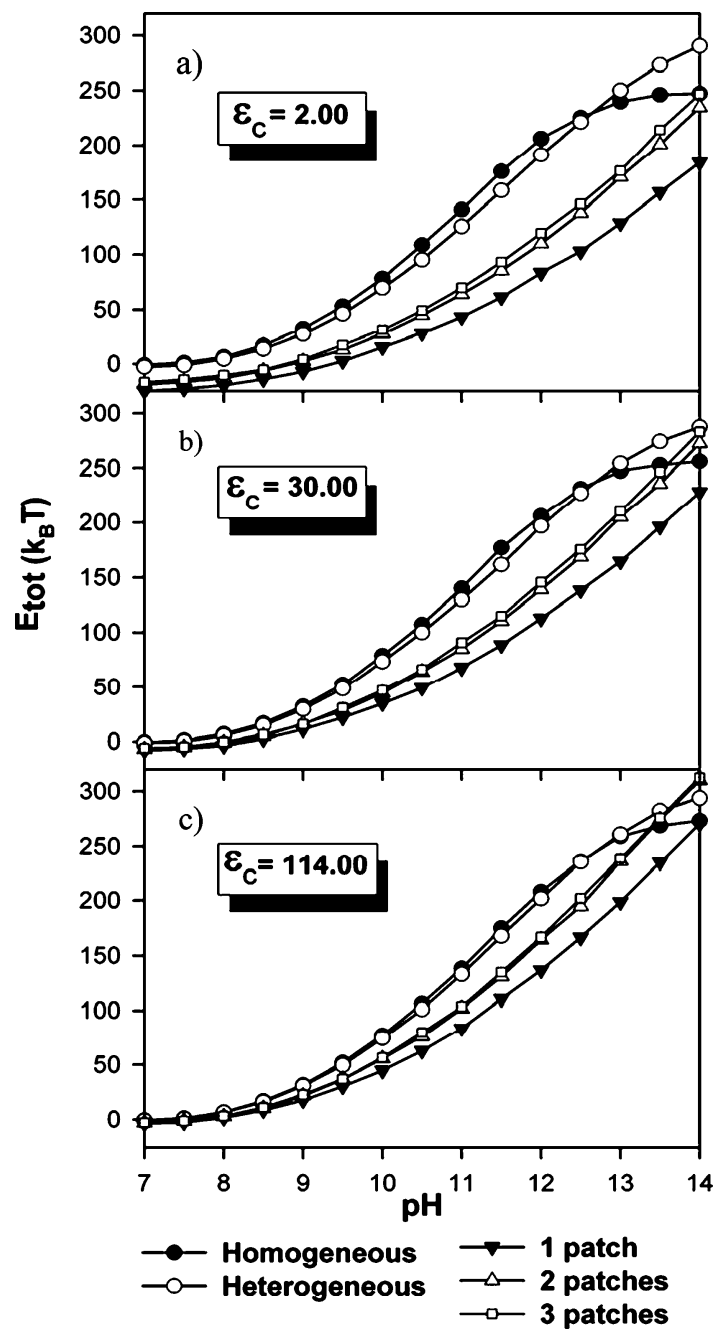

Fig. 9 Effects of Coulomb and reaction field interactions on the total electrostatic energy for various site distributions with $\mathrm{p} K_{\mathrm{a} 1}^{0}=6.0$, $\mathrm{p} K_{\mathrm{a} 2}^{0}=8.0$ and for a NP permittivity equal to (a) $\varepsilon_{\mathrm{c}}=2.0$, (b) $\varepsilon_{\mathrm{c}}=30.0$ and (c) $\varepsilon_{\mathrm{c}}=114.0$.

were performed including ampholytic reaction equilibriums (4) and (5), with $\mathrm{p} K_{\mathrm{a} 1}^{0}<\mathrm{p} K_{\mathrm{a} 2}^{0}$. For patch distributions, charge depletion is observed at the center of patch; charges accumulate at the perimeter where the charge density is lower. This effect is not influenced by the value of the dielectric discontinuity. Nevertheless, charging processes of patch distributions are influenced by the dielectric discontinuity. This influence is the result of a subtle balance between the Coulomb and reaction field interactions. At small separation distances, the relative importance of the Coulomb interactions is more pronounced than the reaction field interactions. This specifically modifies the patch charging process because the number of short distances outweighs the number of long distances, and explains that the total charge of the NP increases when the relative permittivity of the NP $\left(\varepsilon_{\mathrm{c}}\right)$ increases.

For homogeneous and heterogeneous distributions, three different zones of energy variation corresponding to different charging processes steps are observed: (i) When $\mathrm{p} K_{\mathrm{a} 1}^{0}<\mathrm{pH}<$ $\mathrm{p} K_{\mathrm{a} 2}^{0}$ (zone A), the total electrostatic energy is close to $0 k_{\mathrm{B}} T$ for 


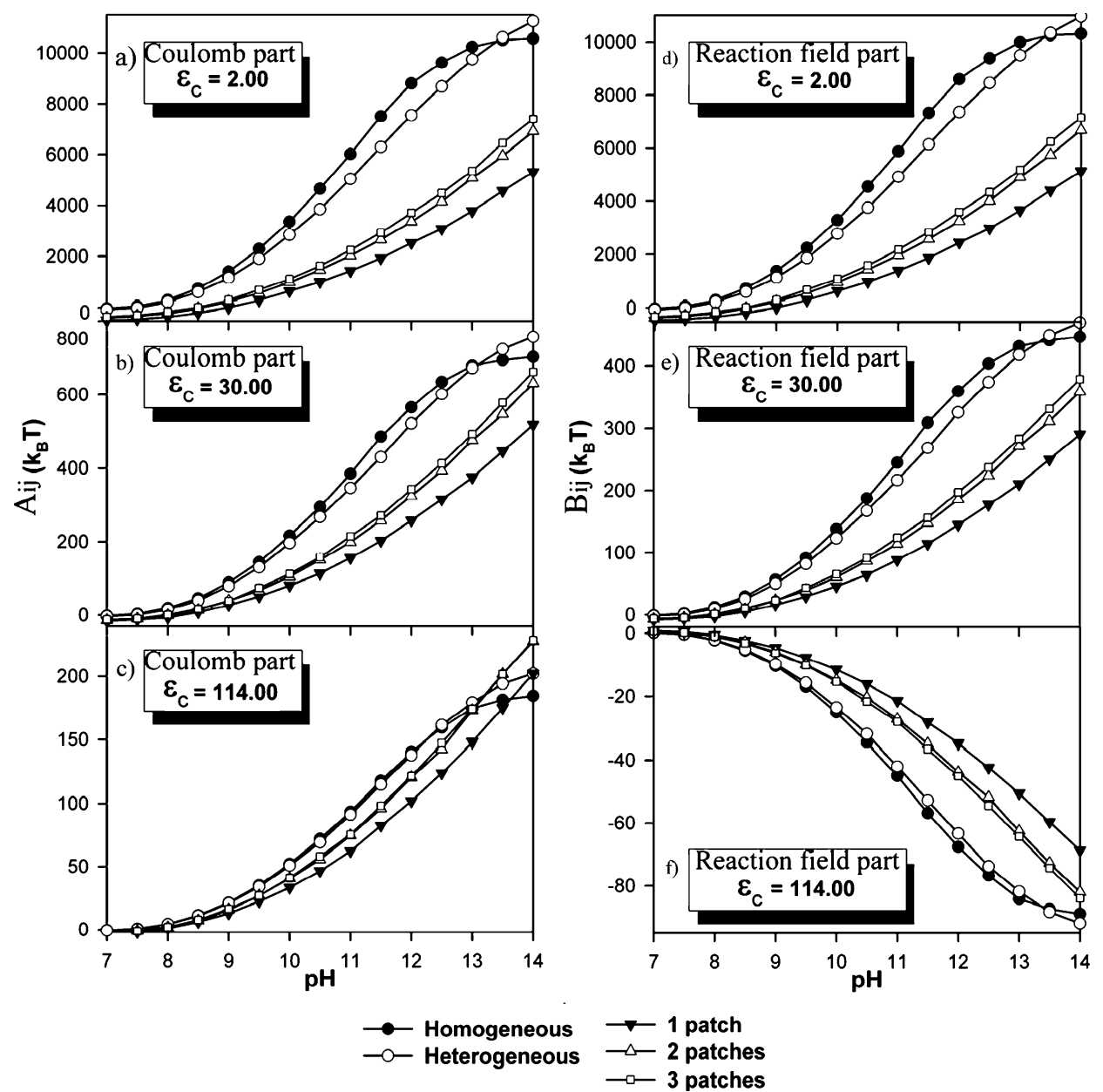

Fig. 10 Detailed Coulomb and reaction field interactions at $T=298 \mathrm{~K}$ and with $\mathrm{p} K_{\mathrm{a} 1}^{0}=6.0, \mathrm{p} K_{\mathrm{a} 2}^{0}=8.0$, for different site distributions, with (a) and (d) $\varepsilon_{\mathrm{c}}=2.0$, (b) and (e) $\varepsilon_{\mathrm{c}}=30.0$ and (c) and (f) $\varepsilon_{\mathrm{c}}=114.0$.

both homogeneous and heterogeneous distributions. This corresponds to a non-charged state if $\Delta \mathrm{p} K_{\mathrm{a}}$ is large enough, and thus to a zero point charge at $\mathrm{pH}=\left(\mathrm{p} K_{\mathrm{a} 1}^{0}+\mathrm{p} K_{\mathrm{a} 2}^{0}\right) / 2$ and to an isoelectric point at the same $\mathrm{pH}$, if $\Delta \mathrm{p} K_{\mathrm{a}}$ is small enough, due to the coexistence of positively and negatively charged sites with non charged states. (ii) When $\left(\mathrm{p} K_{\mathrm{a} 1}^{0}-\delta\right)<\mathrm{pH}<\mathrm{p} K_{\mathrm{a} 1}^{0}$, or $\mathrm{p} K_{\mathrm{a} 2}^{0}$ $<\mathrm{pH}<\left(\mathrm{p} K_{\mathrm{a} 2}^{0}+\delta\right)$ (zone $\left.\mathrm{B}\right)$, with $\delta$ a critical transition parameter of the charging process, the energy of the heterogeneous distribution becomes lower than the homogeneous distribution, with a total charge of heterogeneous distribution smaller than the homogeneous distribution due to the too high energy destabilization in this $\mathrm{pH}$ domain for the charging of the small distance sites found in heterogeneous distributions. (iii) When $\mathrm{pH}<\left(\mathrm{p} K_{\mathrm{a} 1}^{0}-\delta\right)$ or $\mathrm{pH}>\left(\mathrm{p} K_{\mathrm{a} 2}^{0}+\delta\right)$ (zone C), the energy corresponding to the heterogeneous distribution becomes greater than the homogeneous case (at a given $\mathrm{pH}$ ) due to the charging process of the heterogeneous distribution so as to attain a surface charge equal to that of the homogeneous distribution. We demonstrated that $\delta$ is influenced by the distance polydispersity index, $P_{\mathrm{I}}$, of the heterogeneous distribution, but not by the variations in $\varepsilon_{\mathrm{c}}$, due to the fact that no effect of the dielectric discontinuity for the homogeneous and heterogeneous distributions on the charging process is observed.
The present model shows that the distribution of sites plays a crucial role in the charging process and shows the importance of competing interactions between Coulomb interactions and the reaction field. This model represents a useful tool to understand the charging process of NPs induced by an explicit charging process of titration sites. This model can be extended to the study of multiple reactions of titration sites (more than two), heterogeneous distributions of $\mathrm{p} K_{\mathrm{a}}$ and to the charging behaviour of NPs in concentrated solution, including external NPs interaction effects. Studies of the size effect of NPs on the charging behavior and the ionic strength of a surrounding electrolytic solution on this system are currently in progress.

\section{Acknowledgements}

The authors are grateful to Prof. M. Borkovec, Dr P.-Y. Morgantini, Dr A. Laguecir, Dr D. Kony, Dr A. Dejaegere, Dr N. Parthasarathy, F. Alvaltroni and F. Cerveau for their help and encouragement. We gratefully acknowledge the financial support received from the Swiss National Science Foundation (Project No. 200020-101974/1). 


\section{References}

1 D. E. Yates, S. Levine and T. W. Healy, J. Chem. Soc., Faraday Trans. 1, 1974, 70, 1807-1818.

2 D. Chan, J. W. Perram, L. R. White and T. W. Healy, J. Chem. Soc., Faraday Trans. 1, 1975, 71, 1046-1057.

3 J. A. Davis, R. O. James and J. O. Leckie, J. Colloid Interface Sci., 1978, 63, 480-499.

4 J. A. Davis and J. O. Leckie, J. Colloid Interface Sci., 1978, 67, 90-107.

5 T. Hiemstra, W. H. Van Riemsdijk and G. H. Bolt, J. Colloid Interface Sci., 1989, 133, 91-104.

6 T. Hiemstra, J. C. M. De Wit and W. H. Van Riemsdijk, J. Colloid Interface Sci., 1989, 133, 105-117.

7 M. Borkovec, Langmuir, 1997, 13, 2608-2613.

8 M. Borkovec, J. Daicic and G. J. M. Koper, Physica A, 2001, 298, $1-23$.

9 F. H. Stillinger, Jr, J. Chem. Phys., 1961, 35, 1584-1589.

10 C. Tanford and J. G. Kirkwood, J. Am. Chem. Soc., 1957, 79, 5333-5339.

11 C. Tanford, J. Am. Chem. Soc., 1957, 79, 5340-5347.

12 C. Tanford, Physical Chemistry of Macromolecules, John Wiley and Sons, New York, London, 1963.

13 N. Metropolis, A. W. Rosenbluth, M. N. Rosenbluth, A. H. Teller and E. Teller, J. Chem. Phys., 1953, 21, 1087-1092.

14 K. Nagashima and F. D. Blum, J. Colloid Interface Sci., 1999, 217, $28-36$.

15 S. Ross and J.-P. Olivier, On Physical Adsorption, Wiley Interscience, New York, 1954.

16 J. M. Cases and B. Mutaftschiev, Surf. Sci., 1968, 9, 57-72.

17 W. H. Orttung, J. Phys. Chem., 1968, 72, 4066-4071.

18 L. J. Henderson, Am. J. Physiol., 1908, 21, 427-448.

19 K. A. Hasselbach, Biochem. Z., 1916, 78, 112-114.

20 J. T. G. Overbeek, Bull. Soc. Chim. Belg., 1948, 57, 252-261.

21 J. J. Thomson, Philos. Mag., 1904, 7, 237-265.

22 L. T. Wille, Nature, 1986, 324, 46-48.

23 J. de Luca, S. B. Rodrigues and Y. Levin, Europhys. Lett., 2005, 71, 84-90.

24 E. L. Altschuler and A. Perez-Garrido, Phys. Rev. E, 2005, 71, 047703/047701-047703/047704.

25 J. N. Israelachvili, Intermolecular and Surface Forces, Academic Press, London, UK, 1992. 